\title{
INTERACCIÓN E IGUALDAD. LA INTERPRETACIÓN DIALÓGICA DE LA TEORÍA CONSTRUCTIVA DE TIPOS
}

\author{
SHAHID RAHMAN \\ Département de Philosophie \\ CNRs, UMR 8163: STL \\ Université de Lille \\ shahid.rahman@univ-lille3.fr \\ Nicolas Clerbout \\ CDHACS/Instituto de Filosofía \\ Universidad de Valparaíso \\ nicolas.clerbout@uv.cl \\ JUAN REDMOND \\ Instituto de Filosofía \\ Universidad de Valparaíso \\ juan.redmond@uv.cl
}

RESUMEn: Mediante un estudio dialógico de la noción de igualdad definicional de la teoría constructiva de tipos (TCT), se mostrará que tal igualdad, que en la TCT provee el criterio de identidad asociado a un tipo, puede comprenderse, desde el punto de vista lúdico, como resultado de una forma específica de interacción dialógica regida por la regla formal, más conocida recientemente como la regla socrática, que prescribe el uso de jugadas de espejo (copy-cat moves). Esto se ilustrará con el desarrollo dialógico de las partes esenciales de la demostración del axioma de elección de Per Martin-Löf, quien basó su prueba en la igualdad definicional entre la función que prueba el antecedente y la función que ocurre en el consecuente.

PALABRAS CLAVE: identidad, teoría de juegos, regla socrática, jugada de espejo, interlocutor implícito

SUMMARY: The main aim of the present paper is to show, by means of a dialogical study, that the notion of judgemental equality of Constructive Type Theory (CTT), which in this theory furnishes the criterion of identity of a type, can be understood from the game-theoretical point of view as the result of a specific form of dialogical interaction governed by the development rule known as formal rule or (more recently) Socratic rule, which prescribes the use of copy-cat moves. It will be shown, as an example, how to develop the core of a winning strategy for Per Martin-Löf's demonstration of the Axiom of Choice, who based his proof in the judgemental equality between the function in the antecedent and the function occurring in the consequent of the axiom.

KEY WORDS: identity, game theory, Socratic rule, copy-cat moves, implicit interlocutor 


\section{Introducción}

\subsection{Objetivos}

El objetivo principal de este artículo es proveer elementos de análisis que vinculen la noción de interacción argumentativa, concebida dentro de un cuadro dialógico para la lógica constructiva, con la noción de igualdad definicional de la teoría constructiva de tipos (en adelante, TCT) de Per Martin-Löf. Dicho con mayor precisión, intentaremos mostrar que tal igualdad, que en la TCT provee el criterio de identidad asociado a un tipo, puede comprenderse, desde el punto de vista lúdico, como resultado de una forma específica de interacción dialógica regida por la regla conocida como regla formal, más recientemente llamada regla socrática, la cual prescribe el uso de jugadas de espejo (copy-cat moves). Ilustraremos nuestra tesis con el desarrollo dialógico de las partes esenciales de la demostración del axioma de elección de Per Martin-Löf, quien basó su prueba en la igualdad definicional entre la función que prueba el antecedente y la función que ocurre en el consecuente.

\subsection{Antecedentes y marco conceptual}

A partir de una sugerencia de Göran Sundholm, se realizó una lectura dialógica de las reglas de introducción y de eliminación de la deducción natural propuesta por Rahman, Clerbout y Keiff (2009). La idea subyacente es, por una parte, concebir las reglas de introducción como prescripciones que establecen las condiciones que debe satisfacer el proponente $(\mathbf{P})$ para la construcción de una estrategia ganadora y, por la otra, concebir las reglas de eliminación como prescripciones que determinan cómo construir una estrategia ganadora para $\mathbf{P}$ a partir de las afirmaciones que el oponente $(\mathbf{0})$ está forzado a conceder. Esa interacción normativa y armónica de derechos y obligaciones motivó a Clerbout y Rahman (2015) a explorar la contribución de la interpretación dialógica a los fundamentos pragmáticos de la significación en un cuadro constructivista extendido como el de la TCT. $^{1}$

Un nuevo impulso a estas investigaciones provino de una reciente serie de conferencias de Per Martin-Löf en París (2015), quien utilizó la perspectiva dialógica sobre la normatividad de la lógica para

\footnotetext{
${ }^{1}$ Véanse también Rahman y Clerbout 2013, y 2014; Rahman y Redmond 2015; Rahman, Clerbout y McConaughey 2014; Rahman, Clerbout y Jovanovic 2015, y 2016.
} 
salir de una circularidad que parecía amenazar la explicación de las nociones de inferencia y demostración.

En efecto, desde el punto de vista constructivista, una demostración se puede explicar como una cadena de inferencias (inmediatas) a partir de ninguna premisa. Que una inferencia como

$$
\frac{J_{1} \ldots J_{n}}{J}
$$

sea válida significa que la conclusión, el juicio $J$, puede hacerse evidente bajo la suposición de que "conozcamos" $J_{1} \ldots J_{n}$. Esta noción de suposición, que Sundholm llama "suposición epistémica", permite así la explicación de la noción de inferencia válida. No podemos, sin embargo, entender "conocido" en el sentido de demostrado, ya que entonces estamos explicando la noción de inferencia en términos de demostración, mientras que la demostración se ha explicado en términos de inferencia. Martin-Löf sugiere que aquí debemos entender "conocido" como "afirmado en el sentido dialógico". De este modo, los supuestos epistémicos expresan juicios que otro ha llevado a cabo: el oponente. Éste se hace así responsable de las obligaciones contraídas por su propia afirmación. Que la inferencia sea válida significa, entonces, que dado que otro ha asumido la responsabilidad de las premisas, puedo ahora asumir la responsabilidad de la conclusión basándome en la autoridad otorgada al antagonista:

cuando se está dando cuenta de la noción de inferencia inmediata, la noción de demostración no está aún a nuestra disposición. Por ello, la expresión [cuando queremos explicar qué es una inferencia inmediata] "supongamos que $J_{1} \ldots J_{n}$ " nos hace blanco de la acusación de que nuestra explicación es circular [...]. Es ahora de mi parecer que la solución a este problema de circularidad resulta naturalmente de un análisis dialógico [...]. La solución aquí no es suponer que las premisas se conocen en un sentido cualitativo [del término conocer], esto es, que se han demostrado, sino que simplemente debemos suponer que se han afirmado, lo que equivale a decir que otros han tomado la responsabilidad por ellas, y ahora la pregunta que se plantea es si estoy dispuesto a hacerme responsable de la conclusión. [...] Ésta parece ser la definición apropiada de la noción de supuesto epistémico en el sentido de Sundholm. ${ }^{2}$

${ }^{2}$ Transcripción de Ansten Klev de la charla de Martin-Löf en París en mayo de 2015; la traducción es de los autores del presente artículo. Para otro ángulo de reflexión sobre la dialógica y la normatividad, véase Peregrin 2014, pp. 228-229. 
Esta nueva perspectiva llevó a los autores del presente estudio a iniciar una indagación más profunda de los lazos entre el concepto de explicación de significado de la TCT y la concepción pragmática del significado del marco dialógico. ${ }^{3}$ La idea es enfocar el estudio de la conexión entre estas dos teorías del significado en el proceso de constitución de un tipo. En efecto, dado que una de las etapas fundamentales en el proceso de constitución de un tipo es el criterio de identidad definicional, parece natural preguntarse cómo se relaciona un criterio tal de identidad con la interpretación dialógica. La propuesta principal de nuestro artículo es que si vinculamos la regla socrática con la igualdad definicional y con la noción de supuesto epistémico, obtendremos una nueva vía, simple y directa, para utilizar el enfoque de la TCT dentro del marco dialógico. En general, la idea rectora es que la regla socrática es la que subyace tras la interpretación normativa de la noción de supuesto epistémico sugerida por Martin-Löf. Nuestra propuesta puede resumirse de la siguiente manera:

1. O puede atacar un objeto lúdico $b$ que $\mathbf{P}$ ha propuesto como defensa de una proposición elemental $A$.

2. La defensa de un ataque a $b$ consiste en afirmar una igualdad definicional reflexiva o no reflexiva. En ambos casos esta igualdad expresa que el objeto lúdico en cuestión $b$ es igual al que $\mathbf{O}$ usó para defender $A$. La igualdad codifica el resultado de una interacción mediante la cual $\mathbf{P}$ copia jugadas del adversario a fin de proveer las unidades semánticas básicas de la tesis en cuestión.

El caso no reflexivo expresa el caso en el que $\mathbf{0}$ elige $b$ como resolución de una instrucción lúdica (un operador que no tiene la forma argumentativa canónica requerida por $A$ ), que forma parte de una de las concesiones asociadas a la afirmación de la tesis.

De estas consideraciones se sigue la organización del presente trabajo: empezaremos con una discusión del tratamiento de la igualdad en la TCT y luego presentamos su desarrollo en el marco dialógico.

\footnotetext{
${ }^{3}$ La concepción del significado de la TCT se basa en lo que ha sido llamado explicación de significado (meaning explanation) que Martin-Löf 1984, 1996 propone como una alternativa al abordaje idealista de los primeros intuicionistas; véanse Sundholm 1983, y 1986.
} 


\section{Igualdad definicional en la teoría constructiva de tipos}

2.1. Algunos postulados relevantes de la teoría constructiva de tipos En la TCT, las constantes lógicas se interpretan a través de la correspondencia Curry-Howard entre proposiciones y conjuntos. Una proposición se interpreta como un conjunto cuyos elementos representan las pruebas de la proposición. También es posible visualizar un conjunto como la descripción de un problema y sus elementos como las soluciones al problema de una manera similar a la explicación de Kolmogorov del cálculo proposicional intuicionista. Además, en TCT los conjuntos se entienden también como tipos, de modo tal que las proposiciones pueden verse como datos o tipos de prueba. ${ }^{4}$

El objetivo filosófico fundamental de la TCT es desarrollar un enfoque de interpretación total (fully interpreted approach), ${ }^{5}$ donde se presta especial atención a:

evitar mantener separados el contenido y la forma. En su lugar mostraremos ciertas formas de juicio e inferencia que se utilizan en las pruebas matemáticas y al mismo tiempo daremos una explicación semántica de tales formas de juicio. De esta manera, hacemos explícito lo que se suele dar por sentado implícitamente. ${ }^{6}$

En relación con la tarea de explicitación, se trata de poner en el nivel del lenguaje objeto características que determinan el significado y que se formulan usualmente en el nivel meta. De acuerdo con el punto de vista lógico de la TCT, las premisas y la conclusión de una inferencia lógica no son proposiciones, sino juicios:

Una regla de inferencia se justifica explicando la conclusión bajo el supuesto de que las premisas se conocen. Por lo tanto, antes de que una regla de inferencia pueda ser justificada, debe explicarse qué es lo que hay que saber para tener el derecho de hacer un juicio sobre cualquiera de las diversas formas que las premisas y la conclusión pueden tener. ${ }^{7}$

Otros dos principios básicos de la TCT son los siguientes:

\footnotetext{
${ }^{4}$ Véanse Nordström, Petersson y Smith 1990, y Granström 2011.

${ }^{5}$ Véanse Sundholm 1983, 1986, 1997, 2001 y 2013.

${ }^{6}$ Martin-Löf 1984, p. 3, la traducción es nuestra.

${ }^{7}$ Martin-Löf 1984, p. 4, la traducción es nuestra.
} 
- Ninguna entidad sin tipo

- Ningún tipo sin identidad

En consecuencia, podemos tomar la afirmación de que un individuo es un elemento del conjunto $A$ como la afirmación de que dicho individuo ejemplifica el tipo $A$. Un conjunto se define en la TCT especificando sus elementos canónicos (que no se definen por medio de otros elementos) y sus elementos no canónicos, de los que se puede mostrar, usando algún método prescrito de transformación, que son iguales (en este conjunto) a uno canónico. Esto último es lo que prescribe el segundo principio básico y que, en otras palabras, consiste en la introducción de una relación de equivalencia en un conjunto. Así, si $A$ es un tipo y tenemos un objeto $b$ que satisface las condiciones correspondientes, entonces $b$ es un objeto de tipo $A$. Esto se escribe formalmente $b: A .^{8}$

En consecuencia,

$$
b: A
$$

A true

Y se pueden leer como:

$b$ es una prueba de la proposición $A$

$b$ es un elemento del conjunto $A$

$b$ satisface las expectativas de $A$

$b$ es una solución al problema $A$
$A$ es verdadera

$A$ tiene un elemento

$A$ es satisfecha

$A$ tiene una solución

Es esencial distinguir entre el elemento de prueba $b$ (proof-object), el tipo $A$ y el juicio $b: A$, que establece, en este ejemplo, que $b$ es un elemento de prueba para la proposición $A$ (si $A$ es del tipo proposición). En lógica estándar, la existencia de una prueba para una proposición dada se expresa en el nivel del metalenguaje. El hecho de que exista algo (un elemento) $b$ que fundamenta la proposición de que Primus le debe 100 monedas a Secundus (lo que supondría la afirmación correspondiente) se da en el análisis habitual en el nivel del metalenguaje. En la TCT, el fundamento de una afirmación se formula en el nivel de lenguaje objeto por medio de la afirmación de que hay un elemento de prueba de la proposición correspondiente.

${ }^{8}$ Martin-Löf usa el signo " $\in$ " con el fin de indicar que algo, por ejemplo $a$, es de tipo $B$; incluso sugiere que se puede entender como la cópula "es". En Nordström, Petersson y Smith 1990, también se hace uso de esta notación, mientras que otros, como Ranta 1994, utilizan los dos puntos “:”. Granström 2011 distingue los dos puntos de la épsilon: aplica aquéllos a los elementos no canónicos y ésta a los canónicos. Nosotros utilizaremos los dos puntos. 
Siempre que en la TCT se introduce una nueva expresión, se hace por medio de lo que se llama una explicación semántica. En el caso de la introducción de un nuevo tipo, la explicación semántica consiste en (1) describir sus objetos canónicos, (2) proporcionar un algoritmo para reconocer si un objeto no canónico es o no de ese tipo, y (3) dar las condiciones que permitan establecer (o no) la igualdad de dos objetos respecto de ese tipo. El punto 3 se entiende como la tarea de definir una relación de equivalencia apropiada. De este modo, aserciones de la forma $a=b: A$, afirman que los dos objetos $a$ y $b$ satisfacen la relación de equivalencia definida para el tipo $A$. La afirmación $a=b: A$ también se denomina afirmación de igualdad definicional, dado que mediante ella se introducen definiciones explícitas; por ejemplo, de funciones (véase la sección 3.2). Una igualdad tal se transmite entonces por reflexividad, simetría y transitividad, y por sustitución de iguales definicionales ( $c f r$. Ranta 1994, p. 52).

Por obvias razones de espacio, no haremos aquí una descripción detallada y precisa de la TCT. En la próxima sección nos limitaremos a transmitir lo que es pertinente para alcanzar los objetivos del presente trabajo examinando la conjunción y una versión simplificada de las reglas para el existencial. Véase más información sobre esta perspectiva en Martin-Löf (1984, 1996); Ranta (1988, 1994); Nordström, Petersson y Smith (1990); Sundholm (1983, 1986, 1997, 1998, 2009, y 2013). Véase también Granström (2011).

2.2. Las bases de la lógica intuicionista de predicados en el marco de la TCT

\subsubsection{Cuatro tipos de reglas}

Dado que en este marco teórico las proposiciones son conjuntos, los operadores lógicos están definidos como operadores conjuntistas. El significado de tales operadores se establece por medio de cuatro tipos de reglas diferentes, a saber: (i) reglas de formación, (ii) reglas de introducción, (iii) reglas de eliminación y (iv) reglas de igualdad.

(i) Reglas de formación. La inclusión explícita de reglas de formación en un sistema inferencial es una de las características más distintivas de la TCT. Las reglas de formación establecen simultáneamente la sintaxis y los tipos básicos a los que corresponden las constantes lógicas y no lógicas del lenguaje considerado. Más precisamente, las reglas de formación especifican las condiciones bajo las cuales podemos inferir que algo es un tipo (conjunto), y las condiciones que nos permiten señalar que dos tipos (conjuntos) son iguales. 
Por ende, dado que la buena formación incluye no sólo los modos de composición sintáctica, sino también la identificación de los tipos básicos correspondientes, podemos decir que las reglas de formación despliegan al mismo tiempo las reglas de buena formación sintáctica y semántica específicas para un lenguaje determinado.

De hecho, toda demostración TCT $^{9}$ comienza verificando que las expresiones del juicio que se va a demostrar resultan de la aplicación de las reglas de formación correspondientes. Éste es el modo de implementar en el marco de la TCT la idea de un lenguaje totalmente interpretado: cuando leemos una demostración TCT de abajo hacia arriba, en ella se despliegan los elementos sintácticos y semánticos del juicio demostrado.

(ii) Reglas de introducción. Las reglas de introducción, que definen los tipos del sistema, prescriben el modo de formar elementos canónicos y el modo de determinar si dos elementos canónicos son iguales.

(iii) Reglas de eliminación. Establecen el modo de definir funciones (llamados selectores) en el conjunto definido por las reglas de introducción.

(iv) Reglas de igualdad. Como mencionamos en la sección anterior, las reglas de igualdad especifican el modo en el que operan los selectores definidos por las reglas de eliminación y cómo ejecutar su computación dados los elementos canónicos generados por las reglas de introducción.

\subsubsection{Elementos de prueba e igualdad definicional}

El ejemplo más claro de interacción entre proposiciones y elementos de prueba en un sistema de inferencia es el caso de la conjunción. ${ }^{10}$ La proposición $A \wedge B$ (o el conjunto $A \times B$ ) se explica estableciendo que un elemento canónico de $A \wedge B$ es un par de elementos de prueba $(a, b)$, donde $a: A$ y $b: B$, es decir, donde $a$ es un elemento de prueba de $A$, y $b$ de $B$ :

$$
\frac{A: \text { prop } B: \text { prop }}{A \wedge B: \text { prop }} \wedge_{\wedge-\mathrm{F}} \quad \frac{a: A \quad b: B}{(a, b): A \wedge B}{ }_{\wedge \text {-introducción }}
$$

${ }^{9}$ Advertimos al lector que usamos la expresión demostración TCT como forma breve de la expresión demostración en el marco de la teoría constructiva de tipos.

${ }^{10}$ Véase Sundholm 1986. 
Con el fin de definir $\wedge$-eliminaciones vamos a hacer uso de cierto tipo de operadores llamados selectores, a partir del cual se pueden definir nuevas funciones que extraen los componentes que constituyen un elemento de prueba complejo $c$ (por ejemplo, $c=(a, b)$ ). En el caso de la conjunción, los selectores son las funciones de proyección $p$ y $q$ que tienen como valor, respectivamente, el lado izquierdo y el derecho del par de elementos de prueba. Por lo tanto, si $c$ es un elemento de prueba para la conjunción, entonces $p(c)$ nos da el componente izquierdo de $c$ y $q(c)$ su componente derecho.

$$
\frac{c: A \wedge B}{p(c): A} \wedge_{\wedge \text {-p-eliminación }} \frac{c: A \wedge B}{q(c): B} \wedge_{\wedge-q \text {-eliminación }}
$$

Si sabemos que $c=(a, b)$, entonces $p(c)$ restaura el componente izquierdo de $c$ (obtenido por la regla de introducción), esto es: $p(c)=p((a, b))=a$, tal que $a: A$, análogamente $q(c)$ restaura el componente derecho:

$\frac{a: A \quad b: B}{p((a, b))=a: A}{ }_{\wedge \text {-izq- } \beta \text {-igualdad }} \frac{a: A \quad b: B}{q((a, b))=b: B}{ }_{\wedge \text {-der- } \beta \text {-igualdad }}$

Aquí tenemos ejemplos claros de cómo usar la noción de igualdad definicional mencionada líneas antes: las funciones de proyección $p$ y $q$ se definen explícitamente por medio de una regla de inferencia de modo que, dados los elementos de prueba $a$ y $b$, la proyección $q$ de $(a, b)$ es definicionalmente igual a $b$, respecto de la relación de equivalencia que define el tipo $B$, y análogamente se introduce la proyección $p$.

También se puede introducir una regla dual, llamada $\eta$, y que se puede definir de la siguiente manera:

$$
\frac{c: A \wedge B}{(p(c), q(c))=c: A \wedge B}{ }_{\wedge-\eta \text {-igualdad }}
$$

Estas reglas de igualdad definicional, como ha señalado Sundholm (1997, p. 200), no son más que versiones lineales de las etapas de normalización de Prawitz (1965).

Desde el punto de vista del juicio, las reglas del existencial están estrechamente ligadas a los juicios en los que ocurren proposiciones existenciales. En efecto, una forma simplificada de tales reglas da como resultado: 


$$
\begin{aligned}
& (x: A)
\end{aligned}
$$

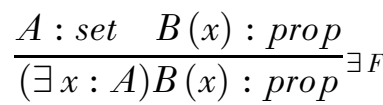

$$
\begin{aligned}
& \frac{a: A \quad b: B(a)}{(a, b):(\exists x: A) B(x)} \exists I
\end{aligned}
$$

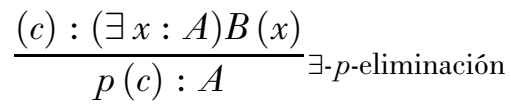

$$
\begin{aligned}
& \frac{(c):(\exists x: A) B(x)}{q(c): B(a)}_{\exists-q \text {-eliminación }} \\
& \frac{a: A \quad b: B(a)}{p((a, b))=a: A^{\exists-i z q-\beta \text {-igualdad }}} \\
& \frac{a: A \quad b: B(a)}{q((a, b))=b: B(a)} \exists-d e r-\beta \text {-igualdad }
\end{aligned}
$$

La diferencia entre el caso proposicional y el caso de la cuantificación reside en la dependencia. En efecto, la regla de formación del existencial muestra que el componente derecho del existencial es una proposición que depende de su lado izquierdo. Dicho con mayor precisión, la parte derecha del existencial es una función proposicional definida sobre el conjunto que constituye su parte izquierda. En otras palabras, mientras que los componentes de una conjunción son tipos independientes entre sí, el segundo componente del existencial es un tipo dependiente del tipo del primer componente.

Un paralelismo similar puede establecerse entre las reglas para el universal y la implicación. ${ }^{11}$

Una vez que las igualdades ocurren en la demostración, se transmiten por medio de la reflexividad, la simetría y la transitividad, y por la sustitución de iguales definicionales. ${ }^{12}$

Como ya anunciamos, no discutiremos aquí el caso de la igualdad proposicional.

A continuación presentaremos una breve introducción a la dialógica estándar, es decir, el marco dialógico para la TCT.

\section{El cuadro dialógico y la teoría constructiva de tipos}

A finales de la década de 1950, Paul Lorenzen creó la lógica dialógica, y luego la desarrolló Kuno Lorenz; ${ }^{13}$ ambos estaban inspirados en la

${ }^{11}$ Véase Martin-Löf 1984, pp. 16-18.

${ }^{12}$ Véase Ranta 1994, p. 52.

${ }^{13}$ Los principales trabajos originales se recogen en Lorenzen y Lorenz 1978. Véase una visión histórica en Lorenz 2001. Otros trabajos se han recogido más recientemente en Lorenz 2008, 2010a, y 2010b. Una relación detallada de los acontecimientos recientes desde Rahman 1993, se puede encontrar en Rahman y Keiff 2005; Keiff 2009, y Rahman 2012. Para la metalógica subyacente, véanse Clerbout 2014a, y 2014b. Para presentaciones en libros de texto, véanse Clerbout 2014b; Redmond 
noción de Wittgenstein de significado como uso. La idea básica del enfoque dialógico de la lógica es que el significado de las constantes lógicas está dado por las normas o reglas para su uso, y estas reglas se entienden como formas específicas de estructuración de la interacción argumentativa. Esta característica argumentativa subyacente en la dialógica a menudo lleva a clasificarla como una teoría pragmática del significado. El enfoque dialógico de la lógica no es una lógica, sino un marco de significado pragmático en el que diferentes lógicas pueden desarrollarse, combinarse y/o compararse. Sin embargo, aquí vamos a limitarnos a las versiones dialógicas de la lógica clásica y la intuicionista.

En un diálogo, dos partes discuten sobre una tesis respetando ciertas reglas fijas. El jugador que afirma la tesis se llama proponente $(\mathbf{P})$; su rival, que pone en tela de juicio la tesis, se llama oponente $(\mathbf{0})$. En su forma original, los diálogos fueron diseñados de tal manera que cada una de las partidas termina, después de un número finito de jugadas, con sólo un jugador ganador. En un diálogo, las acciones o jugadas a menudo se entienden como elocuciones o como actos de habla. En otras palabras, la idea es que las reglas del diálogo no se aplican a las expresiones aisladas del acto de elocución en que fueron proferidas, sino en el contexto del desarrollo de un juego dialógico. Las reglas se dividen en reglas de partículas o reglas para las constantes lógicas (Partikelregeln) y reglas estructurales (Rahmenregeln). Las reglas de partículas regulan las jugadas que constituyen peticiones o requerimientos (a las jugadas del rival) y las que son respuestas (a esas peticiones), mientras que las reglas estructurales determinan el curso general de un juego dialógico (también llamado diálogo).

Son cruciales para el enfoque dialógico los siguientes puntos:

1. La distinción entre significado local (reglas para las constantes lógicas) y significado global (incluido en las reglas estructurales).

2. Las reglas para el significado local, formuladas para jugadores anónimos.

3. La distinción entre el nivel de partida (triunfo de una partida) y el nivel estratégico (existencia de una estrategia ganadora).

y Fontaine 2011, y Rückert 2011. Para estudios de la relación entre la dialógica, la lógica y los juegos, véanse Rahman y Keiff 2010; Rahman y Tulenheimo 2009, y Marion y Rückert 2015. Rahman y sus colaboradores comenzaron recientemente a estudiar el enfoque dialógico de la TCT; véanse Clerbout y Rahman 2015; Rahman y Clerbout 2014, y 2015; Rahman, Clerbout y Jovanovic 2014; Rahman, Clerbout y Jovanovic 2015, y Rahman y Redmond 2016. 
4. Una noción de validez que equivale a una estrategia ganadora para $\mathbf{P}$.

En la sección siguiente presentaremos brevemente la lógica dialógica estándar. La idea es introducir los elementos de la lógica dialógica de una forma autocontenida antes de enriquecerla con los aportes de la TCT.

\subsection{Lógica dialógica estándar}

Sea $\mathbb{L}$ un lenguaje de primer orden construido con base en conectivas proposicionales, cuantificadores, un conjunto numerable de variables individuales, un conjunto numerable de constantes individuales y un conjunto numerable de símbolos de predicado (cada uno con una $n$-idad fija).

Ampliamos el lenguaje $\mathbb{L}$ con dos etiquetas $\mathbf{P}$ y $\mathbf{O}$ que corresponden a los participantes del diálogo y el signo de interrogación "?". Cuando la identidad del jugador no importa (lo que llamamos un jugador anónimo), utilizamos variables $\mathbf{X}$ o $\mathbf{Y}$ (siendo $\mathbf{X} \neq \mathbf{Y}$ ).

Las reglas de partículas proporcionan una descripción abstracta de cómo se procede en el diálogo a nivel local: especifican el modo en el que una proposición puede atacarse o defenderse de acuerdo con su constante lógica principal. Las reglas de partículas son reglas para jugadores anónimos, ${ }^{14}$ en el sentido de que el defensor puede ser tanto $\mathbf{P}$ como $\mathbf{O}$ (por eso también se denominan reglas simétricas). Véase la tabla de la página 61.

La segunda clase de reglas que hemos mencionado, las reglas estructurales, otorgan las condiciones exactas en las que una oración dada genera un juego dialógico. Un juego dialógico para $\varphi$, escrito $\mathrm{D}(\varphi)$, es el conjunto de todas las partidas con $\varphi$ como tesis (véase más adelante la regla de inicio). Las reglas estructurales son las siguientes:

\section{SRO (Regla de inicio)}

Sea $\varphi$ una proposición compleja de $\mathbb{L}$. Para cada $\mathbf{p} \in \mathrm{D}(\varphi)$ tenemos:

$$
\begin{aligned}
& \varrho_{\mathbf{p}}(\mathbf{P}-A)=0, \\
& \varrho_{\mathbf{p}}(\mathbf{O}-n:=i)=1,
\end{aligned}
$$

\footnotetext{
${ }^{14}$ La simetría de la reglas inmuniza el marco dialógico contra conectivas del tipo tonk. Cfr. Rahman y Redmond 2016.
} 
TABLA 1. Reglas de partículas para la dialógica estándar

\begin{tabular}{|c|c|c|c|c|}
\hline Afirmación & $\mathbf{X} ! \varphi \wedge \psi$ & $\mathbf{X} ! \varphi \vee \psi$ & $\mathbf{X} ! \varphi \rightarrow \psi$ & $\mathbf{X} ! \neg \varphi$ \\
\hline \multirow{3}{*}{ Ataque } & $\mathbf{Y} ?_{L}$ & & & \\
& $\mathbf{y} / \mathbf{o}$ & $\mathbf{Y} ?[\varphi, \psi]$ & $\mathbf{Y} ! \varphi$ & $\mathbf{Y} ! \varphi$ \\
& $\mathbf{Y} ?_{R}$ & & & \\
\hline \multirow{3}{*}{ Defensa } & $\mathbf{X} ! \varphi$ & $\mathbf{X} ! \varphi$ & & \\
& $\mathrm{y} / \mathbf{o}$ & $\mathbf{o}$ & $\mathbf{X} ! \psi$ & -- \\
& $\mathbf{X} ! \psi$ & $\mathbf{X} ! \psi$ & & \\
\hline
\end{tabular}

\begin{tabular}{|c|c|c|}
\hline Afirmación & $\mathbf{X} ! \forall x \varphi$ & $\mathbf{X} ! \exists x \varphi$ \\
\hline Ataque & $\mathbf{Y} ?\left[! \varphi\left(x / a_{i}\right)\right]$ & $\mathbf{Y}\left[! \varphi\left(x / a_{1}\right), \ldots, ! \varphi\left(x / a_{n}\right)\right]$ \\
\hline Defensa & $\mathbf{X} ! \varphi\left(x / a_{i}\right)$ & $\mathbf{X} ! \varphi\left(x / a_{i}\right) \operatorname{con} 1 \leq i \leq n$ \\
\hline
\end{tabular}

En ambas tablas, el símbolo "!" anuncia que a continuación sigue una proposición que, si la atacan, debe defenderla el jugador que la plantea. Tenemos, además, que una expresión del tipo $a_{i}$ es una constante individual, y $\varphi\left(a_{i} / x\right)$ expresa la proposición obtenida mediante la sustitución de cada ocurrencia de $x$ en $\varphi$ por $a_{i}$. Cuando una jugada consiste en una pregunta de la forma '? $\left[\varphi_{i}, \ldots, \varphi_{n}\right]$ ' o de la forma $?_{L}, ?_{R}$, entonces el otro jugador elige una proposición entre $\varphi_{i}, \ldots, \varphi_{n}$ y la juega. Así, podemos distinguir - en términos de qué jugador tiene una opción- entre la conjunción y la disyunción, por una parte, y la cuantificación universal y la existencial, por la otra. En los casos de la conjunción y la cuantificación universal, el retador (o atacante) elige la proposición por la cual preguntar. Por el contrario, en los casos de disyunción y cuantificación existencial, el defensor es el único que puede elegir entre varias proposiciones. Obsérvese que no hay defensa en el caso de la regla de partículas para la negación. 
$\varrho_{\mathbf{p}}(\mathbf{P}-m:=j)=2$.

ComentaRio: En otras palabras, cualquier partida $\mathbf{p}$ en $\mathrm{D}(\varphi)$ comienza con $\mathbf{P} ! \varphi$. Llamamos $\varphi$ a la tesis de la partida y del juego dialógico correspondiente. Después de esto, el oponente y el proponente eligen sucesivamente un número entero llamado rango de repetición. El papel de este entero es asegurar que cada partida termine después de un número finito de jugadas.

\section{SR1 (Regla clásica)}

- Toda jugada después de la elección de los rangos de repetición es o bien un ataque, o bien una defensa.

- El rango de repetición elegido por un jugador representa el número máximo de veces que puede desafiar una jugada determinada del adversario o defenderse de ella.

\section{SRli (Regla intuicionista)}

Los jugadores sólo pueden defenderse del último de los ataques que aún no hayan respondido. ${ }^{15}$

SR2 (Regla formal)

El proponente puede afirmar una proposición elemental sólo si $\mathbf{0}$ la afirmó ya antes.

Esta regla es una de las características más sobresalientes de la lógica dialógica y la discutiremos a lo largo de todo el artículo. Como discuten Marion y Rückert (2015), la regla se remonta a la reconstrucción de Aristóteles de la dialéctica platónica: la idea principal es que, cuando una proposición elemental es desafiada (atacada), entonces - desde el punto de vista puramente argumentativo - la única respuesta posible es apelar a las concesiones del oponente (es decir, sin hacer uso de una autoridad más allá de las jugadas realizadas durante la interacción argumentativa). De hecho, podría verse la regla formal como la implementación de un tipo de jugada de espejo (conocida en teoría de juegos como copy-cat strategy): mis razones para afirmar tal proposición son exactamente las mismas que las suyas cuando concedió usted la misma proposición. ${ }^{16}$

${ }^{15}$ Esta cláusula también se conoce como la regla de responda primero a la última obligación (Last Duty First) y permite desarrollar juegos para la lógica intuicionista.

${ }^{16}$ Véanse Rahman, Clerbout y Keiff 2009, y Rahman y Keiff 2010. 
En el caso de juegos en los que se permite que la tesis sea una proposición elemental, hay que reformular la regla formal de la siguiente manera:

\section{SR2* (Regla formal modificada)}

O puede atacar una proposición elemental si y sólo si él mismo aún no la ha afirmado. Sólo el oponente puede atacar proposiciones elementales. El proponente se defiende de un ataque a una proposición elemental mostrando que en el ulterior desarrollo del juego el oponente será forzado a conceder la proposición elemental atacada, digamos en la jugada $n$. En cuanto $\mathbf{0}$ juega $n$, entonces $\mathbf{P}$ se defiende del ataque respondiendo sic $(n)$ (léase: porque tú mismo acabas de conceder en $n$ la misma proposición elemental).

Prosigamos ahora con el resto de las definiciones y reglas estructurales:

- Decimos que una partida es terminal cuando no puede ampliarse en jugadas sucesivas lícitas. Decimos que es $\mathbf{X}$-terminal cuando la última jugada en la partida es una jugada del jugador $\mathbf{X}$.

SR3 (Partida ganada)

El jugador $\mathbf{X}$ gana la partida $\mathbf{p}$ sólo si es $\mathbf{X}$-terminal.

- Una estrategia para un jugador $\mathbf{X}$ en $\mathrm{D}(\varphi)$ es una función que, para cada partida no terminal $\mathbf{p}$, asigna una jugada $M$ a una jugada de $\mathbf{Y}$ que es el último miembro de $\mathbf{p}$, tal que, si extendemos $\mathbf{p}$ con $M$, obtenemos una nueva partida.

- Una estrategia de $\mathbf{X}$ es ganadora en $\mathrm{D}(\varphi)$ si jugando de acuerdo con ella nos lleva a una victoria de $\mathbf{X}$ para $\varphi$ sin importar cómo juegue $\mathbf{Y}$.

La siguiente definición relaciona la estrategia ganadora para $\mathbf{P}$ con validez:

- P tiene una estrategia ganadora para $\varphi$ si y solamente si $\varphi$ es válida en la lógica clásica y/o en la lógica intuicionista jugando de acuerdo con las reglas clásicas y/o intuicionistas. 


\subsection{El marco dialógico para la TCT}

En 1988, Ranta comenzó a explorar la forma de relacionar la TCT con una teoría lúdica del significado para la lógica intuicionista. La idea de Ranta es definir una proposición como el conjunto de sus estrategias ganadoras. En principio la idea es simple y atractiva dado que, para establecer la correspondencia con la TCT, lo que queda por hacer es relacionar la estrategia ganadora con la demostración. Sin embargo, desde el punto de vista inherente a la perspectiva lúdica, reducir el significado a un conjunto de estrategias ganadoras es insatisfactorio. Una estrategia ganadora, como se explicó en los párrafos precedentes, se compone de partidas (plays o games), pero las reglas que determinan el desarrollo de una partida no son reducibles a la función que describe una estrategia ganadora. En otras palabras, es la partida la que provee la base más elemental del significado como interacción y la que determina el concepto dialógico de proposición. Dada esta distinción entre el nivel de partida y el nivel de estrategia, y dado que a la vez queremos implementar la idea de la TCT de asociar una proposición con los elementos que la justifican, parece natural distinguir los objetos lúdicos que se asocian a una proposición en el nivel de partida - tal como en " $p: \varphi$ ", que leemos " $p$ es un objeto lúdico para $\varphi$ "- de los objetos estratégicos asociados a una proposición en el nivel estratégico. Son estos últimos los que corresponden a los elementos de prueba de la TCT. Desde esta perspectiva, el trabajo de Ranta constituye el final y no el principio del proyecto de un cuadro dialógico para la TCT.

A fin de desarrollar un proyecto así, enriquecimos las expresiones que forman parte de un juego dialógico con expresiones de la forma " $p: \varphi$ " (donde a la izquierda de los dos puntos se encuentra un objeto lúdico y a la derecha una proposición), cuyo significado provendrá de reglas locales y estructurales específicas que describirán los modos de composición y análisis de los objetos lúdicos expresados. ${ }^{17}$

La contribución más básica de un objeto lúdico es su contribución a un diálogo material en torno a una proposición elemental. De manera informal: si el jugador $\mathbf{P}$ está dispuesto a afirmar la proposición elemental $A$, es porque el proponente presupone que hay algo, un objeto lúdico, del que $\mathbf{P}$ puede hacer uso para defender $A$, siguiendo una serie de acciones específicas para $A$ prescritas por la regla socrática. En tal forma de diálogos, la regla socrática determina los elementos canónicos y las definiciones (las igualdades definicionales) específicas a cada una de la expresiones elementales en juego.

${ }^{17}$ Véanse Clerbout y Rahman 2015; Rahman y Clerbout 2013, y 2014. 
Es decir, la regla socrática debe redefinirse para cada una de las expresiones elementales en juego; esto produce verdad material.

Por lo tanto, podemos decir que un objeto lúdico prefigura un diálogo material que desarrolla el contenido de la proposición involucrada en una jugada en la que se ha afirmado tal proposición. Esto constituye el origen del enfoque normativo del significado del marco dialógico: el uso (interacción dialógica) debe entenderse como el uso prescrito por una regla que se aplica a expresiones elementales. Esto es lo que Jaroslav Peregrin (2014, pp. 2-3) llama el rol de una expresión lingüistica: según esta terminología, el significado de una proposición elemental se obtiene a partir de su rol en la forma de interacción (específica a esa proposición) prescrita por la regla socrática para el desarrollo de un diálogo material. De esta perspectiva se desprende que los diálogos materiales son importantes no sólo para la cuestión general de la normatividad de la lógica, sino también para la elaboración de un lenguaje con contenido.

Sin embargo, el foco de atención del presente trabajo son los juegos formales. El desarrollo de los diálogos materiales se tratará en un próximo trabajo.

Lo que distingue los diálogos formales de los materiales es que la formulación de la regla socrática en los diálogos formales prescribe una forma de interacción que no sólo permite al proponente basar la afirmación de una proposición elemental en el objeto lúdico propuesto por el oponente (cuando este último postula tal proposición), sino que también permite a $\mathbf{P}$ proceder de la misma manera, cualquiera que sea la proposición elemental arbitraria afirmada por $\mathbf{0}$. En otras palabras, en un diálogo formal la regla socrática no es específica, sino general. Las definiciones que distinguen una proposición de otra se introducen durante el juego. Eso nos conduce a entender los diálogos formales como la forma más pura de razonamiento inmanente a la interacción dialógica.

Así, puesto que en los diálogos formales los objetos lúdicos para las expresiones elementales se remiten a la autoridad de $\mathbf{0}$, lo que necesitamos ahora es describir los objetos lúdicos de la argumentación canónica de las constantes lógicas.

Antes de comenzar a enriquecer el lenguaje del cuadro dialógico estándar con objetos lúdicos, discutiremos primero cómo implementar en dialógica la noción de formación de proposiciones constituidas por constantes lógicas. El resto de nuestro artículo se estructura de la siguiente manera:

- presentación sucinta de las reglas de formación; 
- presentación informal de las reglas locales para el ataque y la defensa de las afirmaciones en las que ocurren objetos lúdicos;

- presentación informal de la reglas estructurales;

- presentación formal de todas las reglas relevantes, y

- presentación de un ejemplo.

\subsubsection{La formación de proposiciones}

En la lógica habitual en general, y también en la dialógica estándar, las reglas que rigen las constantes lógicas presuponen reglas de buena formación. Tales reglas son habitualmente parte del metalenguaje de un sistema lógico determinado. Inspirados por la TCT, queremos introducir aquí reglas en el lenguaje objeto que permitan verificar si determinada expresión está bien formada, y más en particular, si determinada expresión es o no una proposición. Tales reglas son reglas locales que completan el significado local de las unidades mínimas del lenguaje lógico del sistema. Por razones de brevedad, solamente presentaremos una versión condensada. Más aún, nos restringiremos aquí sólo al caso de la formación de constantes lógicas. Para una presentación completa, véase Clerbout y Rahman (2015).

TABLA 2. Reglas de formación

\begin{tabular}{|c|c|c|}
\hline $\begin{array}{l}\text { AFIRMACIÓN } \\
{[\text { mientras que " } \varphi \mathbf{K} \psi "} \\
\text { expresa una conectiva } \\
\text { diádica, } \\
\text { "( } \mathbb{Q} x: A) \varphi(x) " \text { expresa } \\
\text { un cuantificador] }\end{array}$ & $\begin{array}{l}\text { ATAQUE } \\
\text { [en caso de } \\
\text { que haya } \\
\text { varios ataques } \\
\text { posibles, es } \\
\text { el atacante el } \\
\text { que elige] }\end{array}$ & DEFENSA \\
\hline $\mathbf{X} ! \varphi \mathbf{K} \psi:$ prop & $\begin{array}{c}\mathbf{Y} ?_{\mathrm{F}^{\mathrm{K}_{1}}} \\
\mathrm{y} / \mathrm{o} \\
\mathbf{Y} ?_{\mathrm{F}^{\mathbf{K}_{2}}}\end{array}$ & $\begin{array}{c}\mathbf{X} ! \varphi: \text { prop } \\
\mathrm{y} / \mathrm{o} \\
\mathbf{X} ! \psi: \text { prop }\end{array}$ \\
\hline $\mathbf{X} !(\mathbb{Q} x: A) \varphi(x):$ prop & $\begin{array}{l}\mathbf{Y} ?_{\mathrm{F}^{\mathbb{Q}_{1}}} \\
\mathrm{y} / \mathrm{o} \\
\mathbf{Y} ?_{\mathrm{F}_{2}}^{\mathbb{Q}_{2}}\end{array}$ & $\begin{array}{l}\mathbf{X} ! A: \text { set } \\
\text { y/o } \\
\mathbf{X} ! \varphi(x): \operatorname{prop}(x: A)\end{array}$ \\
\hline $\mathbf{X} ! \perp:$ prop & -- & -- \\
\hline
\end{tabular}




\subsubsection{Diálogos con objetos lúdicos}

1) Objetos lúdicos y la forma canónica de una argumentación

Además de las reglas de formación, las reglas que proveen el significado local de una afirmación $\pi$ indican las jugadas que determinan la forma canónica de argumentación del objeto lúdico específico a la proposición (o conjunto) en juego en la afirmación $\pi$.

La forma de argumentación canónica incluye la indicación de qué acción dialógica (afirmación o requerimiento) debe ser llevada a cabo por quién (defensor o atacante). Así, la forma de argumentación canónica para la implicación $p: \varphi \supset \psi$ afirmada por $\mathbf{X}$ indica:

a) que el adversario $\mathbf{Y}$ debe afirmar el antecedente (proporcionando al mismo tiempo un objeto lúdico para él) y debe solicitar que $\mathbf{X}$ afirme el consecuente (con un objeto lúdico adecuado); es decir, $\mathbf{Y} ! p_{1}: \varphi, ? ! \psi{ }^{18}$

b) que el defensor, $\mathbf{X}$, debe responder afirmando el consecuente solicitado (con su correspondiente objeto lúdico); es decir, $\mathbf{X} ! p_{2}: \psi$.

En otras palabras, la forma canónica de argumentación para la implicación es la siguiente:

Afirmación: $\mathbf{X} ! p: \varphi \supset \psi$

Ataque: $\mathbf{Y} ! p_{1}: \varphi, ? ! \psi$

Defensa: $\mathbf{X} ! p_{2}: \psi$.

Nótese que en el caso de la conjunción es el defensor quien debe proponer el objeto lúdico para ambas partes de la conjunción, pero es el atacante $\mathbf{Y}$ quien tiene el privilegio de elegir cuál de las partes ha de ser defendida por $\mathbf{X}$. En efecto, la forma canónica de argumentación del objeto lúdico para la conjunción es producto de la siguiente interacción:

$$
\begin{array}{lc|ll}
\multicolumn{4}{c}{\text { Afirmación: } \mathbf{X} ! p: \varphi \wedge \psi} \\
\text { Ataque: } & \mathbf{Y} ?_{L} ! \varphi & \text { Ataque: } \quad \mathbf{Y} ?_{R} ! \psi \\
\text { Defensa: } & \mathbf{X} ! p_{1}: \varphi & \text { Defensa: } \quad \mathbf{X} ! p_{2}: \psi
\end{array}
$$

${ }^{18}$ La notación es una adaptación del formalismo de Keiff 2007, sección 2.3.4, y Clerbout 2014a, y 2014b. 
Las formas canónicas de argumentación de las restantes constantes lógicas son las siguientes:

Afirmación: $\mathbf{X} ! p:(\exists x: A) \varphi$

\begin{tabular}{ll|ll} 
Ataque: & $\mathbf{Y} ?_{L} ! A$ & Ataque: & $\mathbf{Y} ?_{R} ! \varphi\left(p_{1}\right)$ \\
Defensa: & $\mathbf{X} ! p_{1}: A$ & Defensa: & $\mathbf{X} !: p_{2}: \varphi\left(p_{1}\right)$
\end{tabular}

Afirmación: $\mathbf{X} ! p: \varphi \vee \psi$

Ataque: $\mathbf{Y}$ ? $\mathrm{V}$

Defensa: $\mathbf{X} ! p_{1}: \varphi \quad \mid$ Defensa: $\mathbf{X} ! p_{2}: \psi$

Más en general, la forma canónica de argumentación de un objeto lúdico para una constante lógica está determinada (a nivel local) por la tríada:

Afirmación de $\mathbf{X}$, ataque de $\mathbf{Y}$ y defensa de $\mathbf{X}$.

2) Prescripciones y forma argumentativa

La forma canónica de argumentación de un objeto lúdico codifica tanto la prescripción como el resultado de llevar a cabo la prescripción implicada en una afirmación dada. Sin embargo, también podemos determinar el significado local mediante el aislamiento del nivel prescriptivo. Este nivel subraya los compromisos y los derechos que caracterizan el significado de la afirmación en juego: hace explícita lo que llamamos su forma argumentativa.

A fin de expresar la forma argumentativa, introducimos ciertos operadores que llamamos instrucciones, como $L^{\vee}(p), R^{\wedge}(p)$. Así, por ejemplo, la forma argumentativa de la disyunción $p: \phi \vee \psi$ indica que su defensa incluye expresiones tales como $L^{\vee}(p)$ y $R^{\vee}(p)$, llamadas respectivamente instrucción izquierda y derecha de la disyunción. Informalmente pueden leerse como las indicaciones "Elija un objeto lúdico para el componente izquierdo (derecho) de p".

De esto se desprende fácilmente la forma argumentativa del resto de las constantes lógicas. Por ejemplo, la de la implicación es:

Afirmación: $\mathbf{X} ! p: \varphi \supset \psi$

Ataque: $\mathbf{Y} ! L^{\supset}(p): \varphi$

Defensa: $\mathbf{X} ! R^{\supset}(p): \psi$ 
$\mathrm{Si}$ amalgamamos el resultado de la interacción en un solo objeto lúdico (no canónico) compuesto, obtenemos:

$$
\left(L^{\supset}(p), R^{\supset}(p)\right): \varphi \supset \psi
$$

Sin embargo, esto no muestra qué acción fue llevada a cabo ni por quién. Es más, es la secuencia la que hace posible producir el objeto estratégico adecuado. Por ejemplo, la forma argumentativa de $p:(A \wedge B) \supset A$ afirmada por $\mathbf{X}$ es:

Ataque: $\mathbf{Y} ! L^{\supset}(p): A \wedge B$

Defensa: $\mathbf{X} ! R^{\supset}(p): A$

Si hacemos explícita la estructura interna de la forma argumentativa del antecedente, obtenemos:

$$
\begin{aligned}
& \mathbf{Y} !\left(L^{\wedge}\left(L^{\supset}(p)\right), R^{\wedge}\left(L^{\supset}(p)\right)\right): A \wedge B \\
& \mathbf{X} ! R^{\supset}(p): A .
\end{aligned}
$$

Aunque el modo de obtener una victoria no es parte de las reglas locales, las instrucciones determinan cómo concebir ese modo (desde un punto de vista estratégico). En efecto, para ganar una partida que comienza con la afirmación $p:(A \wedge B) \supset A$, la forma argumentativa hace obvio que el defensor - cuando lleva a cabo la instrucción $R^{\supset}(p)$ - debe producir un objeto lúdico que sea idéntico al objeto lúdico que $\mathbf{O}$ elija (sea cual fuere) cuando lleva a cabo la instrucción $L^{\wedge}\left(L^{\supset}(p)\right)$ : A. En otras palabras, el objeto estratégico (en forma simplificada) es la siguiente ecuación que incluye en su notación los jugadores que deben llevar a cabo la instrucción requerida por la forma argumentativa:

$$
\mathbf{P} ! L^{\wedge}\left(L^{\supset}(p)\right)^{\mathbf{0}}=R^{\supset}(p)^{\mathbf{P}}=p_{i}:(A \wedge B) \supset A
$$

O en forma condensada y sin llevar a cabo las instrucciones:

$$
\mathbf{P} ! L^{\wedge}\left(L^{\supset}(p)\right)=R^{\supset}(p):(A \wedge B) \supset A
$$

El llevar a cabo la prescripción indicada por una instrucción requiere dos reglas, a saber:

- Resolución de instrucciones. Establece cómo atacar una instrucción y cómo defenderla mediante la elección de un objeto lúdico. 
- Sustitución de instrucciones. Asegura que si una instrucción determinada ha sido resuelta por medio de la elección de un objeto lúdico, digamos $b$, entonces la misma instrucción será siempre reemplazada por el mismo objeto lúdico $b$ toda vez que la misma instrucción vuelva a ocurrir.

Esto muestra que las instrucciones son funciones predefinidas: ambas despliegan el concepto dialógico de función. En efecto, en dialógica, las funciones son reglas de correspondencia tales que si un jugador juega un elemento de un dominio predeterminado, entonces el contrincante debe relacionarlo con su imagen, de manera que la relación satisfaga la unicidad de la valuación. En el caso particular de instrucciones, el contrincante $\mathbf{Y}$ pedirá llevar a cabo la acción requerida por la instrucción. Por ejemplo, la acción requerida para la defensa de la instrucción $R^{\vee}(p)$ consiste en elegir un objeto lúdico para $R^{\vee}$ dado el argumento $p$. Cuando una acción tal se ha llevado a cabo, decimos que la instrucción ha sido cumplida o llevada a cabo y llamamos a la acción misma resolver una instrucción. Ahora bien, con el objetivo de asegurar la unicidad de la valuación, debemos asegurarnos de que la resolución de instrucciones para un argumento determinado siempre dará la misma valuación. Esto es, si $R^{\vee}(p)$ se ha resuelto una vez con, supongamos, $p_{1}$, siempre que $R^{\vee}(p)$ ocurra durante el juego se debe sustituir del mismo modo, es decir, con $p_{1}$. Ése es el rol de la regla de sustitución de instrucciones.

De hecho, el desarrollo de un diálogo determinado por el razonamiento inmanente incluye cuatro etapas distintas:

1) Formulación explícita de la forma (pura) de argumentación. Es decir, formular de manera explícita las instrucciones que hacen manifiesta la forma argumentativa de la tesis.

2) Resolución de instrucciones. Llevar a cabo las prescripciones indicadas por la forma argumentativa, haciendo explícitos los objetos lúdicos que resultan de seguir las instrucciones indicadas por la forma argumentativa.

3) Establecer la forma canónica de argumentación. Hacer explícito, por medio de igualdades definicionales, que los objetos lúdicos obtenidos en el paso anterior tienen la forma argumentativa canónica adecuada.

4) Producir el objeto estratégico. Producir el objeto estratégico específico a la estrategia ganadora para la tesis. Esto equivale 
a producir el objeto estratégico a partir de los objetos lúdicos producidos en el paso 3.

Mientras que los dos primeros pasos involucran el significado local, el paso 3 concierne al desarrollo de las reglas estructurales, y el paso 4 requiere describir cómo producir una estrategia ganadora. Dado que los pasos 1 y 2 han sido ya discutidos, abordaremos ahora la tercera etapa: establecer la forma canónica. Respecto de la última etapa (producir el objeto estratégico), seremos menos explícitos y nos restringiremos a mostrar cómo implementarla en la demostración del axioma de elección.

\section{Igualdad e interacción}

4.1. La jugada de espejo y el razonamiento inmanente

Como ya mencionamos, una de las características más sobresalientes de la dialógica es la llamada regla formal, la cual prescribe jugadas de espejo. El padre de esta idea es sin duda Platón: ${ }^{19}$

- No hay mejor fundamento para una afirmación en un argumento que una fundamentación inmanente a ese mismo contexto de argumentación. El proponente fundamenta su afirmación apelando a las mismas razones que el oponente concedió durante tal argumentación: no hay otra justificación que las que se desprenden del desarrollo de un proceso dialógico. Esta forma de fundamentación es la que da origen al razonamiento formal. ${ }^{20}$

Subrayemos aquí los siguientes puntos:

1. El razonamiento formal resulta de una forma específica de interacción.

2. El razonamiento formal resultante no puede entenderse aquí como carente de contenido y reducido a una jugada puramente sintáctica.

Ambos puntos son importantes para entender la crítica generada contra el razonamiento formal en general y contra la lógica en particular.

${ }^{19}$ Cfr. Gorgias $472 \mathrm{~b}-\mathrm{c}$.

${ }^{20}$ Trabajos recientes muestran que esta regla también ocupa un lugar central en el desarrollo de los vínculos que llevan de la dialéctica aristotélica a la silogística. Véase Rahman, McConaughey y Crubellier 2016. 
Sólo en el último periodo de la historia de la filosofía el razonamiento formal se ha reducido a manipulación sintáctica — presumiblemente la primera aparición explícita de un enfoque sintáctico de la lógica se debe a Leibniz y su "pensée aveugle" (aunque cabe decir que esta noción de Leibniz no era reductora) - . La noción de razonamiento formal, tanto en Aristóteles como en Platón, no es ni "estática" ni "vacía de significado": la idea que subyace tras esta forma de interacción es que el significado y la justificación de una afirmación es el resultado de lo que se ha presentado durante la interacción argumentativa.

Comencemos nuestro estudio mediante la presentación informal de un ejemplo que muestra cómo introducir la igualdad definicional en el lenguaje objeto a partir de un proceso argumentativo:

Supongamos que el proponente plantea la tesis de que si el oponente concede la conjunción, por ejemplo $A \wedge B$, el proponente será capaz de defender con éxito la afirmación $B \wedge A$. Es decir, $\mathbf{P}$ afirma que posee una estrategia ganadora para la transformación conmutativa de la conjunción. Presentemos muy informalmente el desarrollo dialógico de esta tesis:

1. $\mathbf{0} ! p: A \wedge B \quad$ (concesión)

2. $\mathbf{P} ! q: B \wedge A$

3. $\mathbf{0} ?_{L} \quad$ (el oponente lanza su ataque preguntando por la componente izquierda)

4. $\mathbf{P} ! L^{\wedge}(q): B$

5. $\mathbf{O} L^{\wedge}(q)$ ? $\quad(\mathbf{O}$ pide a $\mathbf{P}$ que resuelva la instrucción eligiendo un objeto lúdico)

6. $\mathbf{P} ?_{R}$. Dado que el objetivo aquí es desarrollar una estrategia ganadora, vamos a suponer que $\mathbf{P}$ hace la jugada más inteligente, y ésta es sin duda lanzar un contraataque: la idea es forzar a que sea $\mathbf{0}$ el primero en elegir un objeto lúdico para $B$. Una vez que $\mathbf{O}$ haya hecho su elección, $\mathbf{P}$ puede simplemente copiar esa elección:

7. $\mathbf{0} ! R^{\wedge}(p): B$

8. $\mathbf{P} R^{\wedge}(q)$ ? $\quad \mathbf{P}$ pide a $\mathbf{0}$ que resuelva la instrucción escogiendo un objeto lúdico para el lado derecho de la conjunción 
9. $\mathbf{0} ! b: B \quad(\mathbf{O}$ resuelve la instrucción eligiendo el objeto lúdico $b)$ Ahora $\mathbf{P}$ tiene la información que necesitaba y copia la elección de $\mathbf{O}$ para contestar el desafío de $\mathbf{O}$ lanzado con la jugada 5:

\section{0. $\mathbf{P} ! b: B$}

Esto provee una de las ramas de la estrategia ganadora. La otra rama está constituida por la serie de jugadas que resultan si $\mathbf{0}$ inicia el juego con un ataque a la derecha. Sin embargo, debe quedar claro que la secuencia es completamente análoga.

Demos ahora un paso más en profundidad haciendo más explícito el uso de la jugada espejo:

- Desde un punto de vista estratégico, $\mathbf{P}$ está considerando el objeto lúdico de la parte derecha de $p$ como definicionalmente equivalente al de la parte izquierda de $q$. En efecto, la estrategia ganadora para $B \wedge A$ está constituida por el par $(b, a)$ tal que $b$ es definicionalmente equivalente a la parte derecha de $A \wedge B$ y $a$ definicionalmente equivalente a su parte izquierda.

$$
\begin{gathered}
\left(R^{\wedge}(p), L^{\wedge}(p)\right): B \wedge A \\
R^{\wedge}(p)=b: B \\
L^{\wedge}(p)=a: A
\end{gathered}
$$

No importa qué objeto lúdico elija para la parte derecha de la conjunción, P lo copiará cuando resuelva la instrucción $L^{\wedge}(q)$ - esta interacción es la que llamamos jugada de espejo-.

La igualdad expresa en forma compacta la interacción que produce la estrategia ganadora. En efecto, si $\mathbf{0}$ resuelve $R^{\wedge}(p)$ con el objeto lúdico $b$, y $\mathbf{P}$ cumple la instrucción $L^{\wedge}(q)$ mientras elige el mismo objeto lúdico, entonces $\mathbf{P}$ no solamente está haciendo uso de la concesión $b: B$ de $\mathbf{0}$, sino que también está eligiendo para $B$ el mismo objeto lúdico que $\mathbf{0}$ había elegido para la resolución de $R^{\wedge}(p)$. Por ello, si se le pidiera a $\mathbf{P}$ justificar $b: B$, podrá responder con la igualdad definicional $R^{\wedge}(p)=b: B$ [léase yo elegí b como justificación de B pues tú mismo justificaste $\mathrm{B}$ con $\mathrm{b}]$.

El punto de vista estratégico es sólo una generalización del procedimiento que se lleva a cabo en el nivel de juego. Por lo tanto, nuestra presentación informal será la siguiente (partimos de la jugada 10, ya que las precedentes permanecen sin cambios): 
10. $\mathbf{P} ! b: B$

11. $\mathbf{0} ?=b$

12. $\mathbf{P} ! R^{\wedge}(p)=b: B$

Este ejemplo concierne a la igualdad definicional entre elementos de un conjunto. Consideremos ahora también el caso de la igualdad proposicional, es decir, la igualdad definicional entre dos proposiciones que son dos instancias del tipo prop. Tomemos, por ejemplo, la tesis de que existe una estrategia ganadora para $p:(\exists x: A) B(x)$ si el oponente concede $q:(\forall x: A) B x$. El nivel de juego que conduce a la constitución de una estrategia ganadora se basa en el hecho de que $\mathbf{P}$ puede elegir, para la resolución de una instrucción, el primer componente del existencial, es decir, un elemento del dominio de cuantificación $A$, un objeto lúdico, por ejemplo $a$, que es definicionalmente igual al elemento elegido por $\mathbf{0}$ para resolver la instrucción de la primera componente del universal. La formulación explícita de este proceso conduce a la igualdad definicional $L^{\forall}(q)=a: A$. Ahora bien, dado que la resolución de $L^{\forall}(q)$ se extenderá a $B\left(L^{\forall}(q)\right)$, obtenemos que $B\left(L^{\forall}(q)\right)$ y $B(a)$ son proposiciones equivalentes, es decir, $L^{\forall}(q)=B(a)$ : prop. Desde el punto de vista estratégico, esta forma de igualdad dará un buen resultado para cualquier elemento de $A$ que $\mathbf{0}$ elija. En nuestro caso, las siguientes igualdades codifican la estrategia ganadora: $L^{\exists}(p)=L^{\forall}(q): B$ y $B\left(L^{\forall}(q)\right)=B\left(L^{\exists}(p)\right)$ : prop.

La introducción de la reflexividad de la igualdad definicional surge como resultado de una jugada de espejo por medio de la cual $\mathbf{P}$ "copia" un objeto lúdico $a$ postulado antes por $\mathbf{0}$ para la misma proposición, tal que cuando $\mathbf{0}$ introduce $a$, lo hace como resultado de uno de los siguientes casos:

1. Introduce $a$ para resolver exactamente la misma resolución que llevó a $\mathbf{P}$ a elegir $a$.

2. Introduce $a$ como parte de una afirmación que es la misma que copia $\mathbf{P}$.

En suma, esto sugiere que la jugada de espejo de la formulación estándar de la dialógica para juegos formales contiene implícitamente dos formas distintas de igualdad definicional: la reflexiva y la no reflexiva. Es el enfoque de la TCT el que permite hacer explícitas 
las diferentes formas de copias puestas en marcha por la jugada de espejo.

4.2. La regla socrática y la igualdad definicional

Las consideraciones precedentes nos llevan a una reformulación de la regla formal cuya idea básica es la siguiente:

- Las expresiones elementales de $\mathbf{P}$ que resultan de la resolución de instrucciones pueden ser atacadas por $\mathbf{0}$.

- La respuesta a estos ataques consiste en la afirmación de una igualdad definicional. $\mathrm{Y}$ se distinguen los casos reflexivos y no reflexivos mencionados en la discusión precedente.

- De hecho, una igualdad definicional afirmada por $\mathbf{P}$ expresa la igualdad entre un objeto lúdico introducido por $\mathbf{0}$ y una instrucción también introducida por $\mathbf{0}$.

En la subsección siguiente presentaremos por medio de tablas (simplificadas) las reglas que acabamos de discutir. En la sección 5 ilustraremos cómo aplicarlas. 
4.3. Las reglas de juego

4.3.1. Significado local

Forma canónica de argumentación

\begin{tabular}{|l|l|l|}
\hline AFIRMACIÓN & ATAQUE & DEFENSA \\
\hline $\mathbf{X} ! p:(\exists x: A) \varphi$ & $\begin{array}{l}\mathbf{Y} ?_{L} ! A \\
\text { o } \\
\mathbf{Y} ?_{R} ! \varphi\end{array}$ & $\begin{array}{l}\mathbf{X} ! p_{1}: A \\
\text { respectivamente } \\
\mathbf{X} ! p_{2}: \varphi\left(p_{1}\right)\end{array}$ \\
\hline $\mathbf{X} ! p: \varphi \wedge \psi$ & $\begin{array}{l}\mathbf{Y} ?_{L} ! \varphi \\
\text { o } \\
\mathbf{Y} ?_{R} ! \psi\end{array}$ & $\begin{array}{l}\mathbf{X} ! p_{1}: \varphi \\
\text { respectivamente } \\
\mathbf{X} ! p_{2}: \psi\end{array}$ \\
\hline $\mathbf{X} ! p:(\forall x: A) \varphi$ & $\mathbf{Y} ! p_{1}: A, ? ! \varphi$ & $\mathbf{X} ! p_{2}: \varphi\left(p_{1}\right)$ \\
\hline $\mathbf{X} ! p: \varphi \supset \psi$ & $\mathbf{Y} ! p_{1}: \varphi, ? ! \psi$ & $\mathbf{X} ! p_{2}: \psi$ \\
\hline $\begin{array}{l}\mathbf{X} ! p: \neg \varphi \\
\text { También expresa- } \\
\mathbf{X} ! p: \varphi \supset \perp\end{array}$ & $\mathbf{Y} ! p_{1}: \varphi, ? ! \perp$ & $\begin{array}{l}\mathbf{X} ! \perp \\
(\mathrm{el} \text { jugador } \mathbf{X} \\
\text { abandona) }\end{array}$ \\
\hline $\mathbf{X} ! p: \varphi \vee \psi$ & $\mathbf{Y} ? \vee[! \varphi \mid ! \psi]$ & $\begin{array}{l}\mathbf{X} ! p_{1}: \varphi \\
\text { o } \\
\mathbf{X} ! p_{2}: \psi\end{array}$ \\
\hline
\end{tabular}


Forma argumentativa

\begin{tabular}{|c|c|c|}
\hline AFIRMACIÓN & ATAQUE & DEFENSA \\
\hline $\mathbf{X} ! p:(\exists x: A) \varphi$ & $\begin{array}{c}\mathbf{Y} ?_{L} \\
\mathbf{0} \\
\mathbf{Y} ?_{R}\end{array}$ & $\begin{array}{l}\mathbf{X} ! L^{\exists}(p): A \\
\text { respectivamente } \\
\mathbf{X} ! R^{\exists}(p): \varphi\left(L^{\exists}(p)\right)\end{array}$ \\
\hline $\mathbf{X} ! p:\{x: A \mid \varphi\}$ & $\begin{array}{c}\mathbf{Y} ?^{L} \\
\mathbf{o} \\
\mathbf{Y} ?_{R}\end{array}$ & $\begin{array}{l}\mathbf{X} ! L^{\{\cdots\}}(p): A \\
\text { respectivamente } \\
\mathbf{X} ! R^{\{\cdots\}}(p): \varphi\left(L^{\{\cdots\}}(p)\right)\end{array}$ \\
\hline $\mathbf{X} ! p: \varphi \wedge \psi$ & $\begin{array}{c}\mathbf{Y} ?_{L} \\
\mathbf{O} \\
\mathbf{Y} ?_{R}\end{array}$ & $\begin{array}{l}\mathbf{X} ! L^{\wedge}(p): \varphi \\
\text { respectivamente } \\
\mathbf{X} ! R^{\wedge}(p): \psi\end{array}$ \\
\hline $\mathbf{X} ! p:(\forall x: A) \varphi$ & $\mathbf{Y} ! L^{\forall}(p): A$ & $\mathbf{X} ! R^{\forall}(p): \varphi\left(L^{\forall}(p)\right)$ \\
\hline $\mathbf{X} ! p: \varphi \supset \psi$ & $\mathbf{Y} ! L^{\supset}(p): \varphi$ & $\mathbf{X} ! R^{\supset}(p): \psi$ \\
\hline $\begin{array}{l}\mathbf{X} ! p: \neg \varphi \\
\text { También expresado } \\
\text { como } \\
\mathbf{X} ! p: \varphi \supset \perp\end{array}$ & $\begin{array}{l}\mathbf{Y} ! L^{\neg}(p): \varphi \\
\mathbf{Y} ! L^{\supset}(p): \varphi\end{array}$ & $\begin{array}{l}\mathbf{X} ! R^{\neg}(p): \perp \\
\mathbf{X} ! R^{\supset}(p): \perp\end{array}$ \\
\hline $\mathbf{X} ! p: \varphi \vee \psi$ & $\mathbf{Y} ? \vee$ & $\begin{array}{l}\mathbf{X} ! L^{\vee}(p): \varphi \\
\mathbf{X}: R^{\vee}(p): \psi\end{array}$ \\
\hline
\end{tabular}

Sustitución de Afirmaciones Dependientes (Sust-AD) ${ }^{21}$

\begin{tabular}{|l|l|l|}
\hline $\mathbf{X} ! \pi\left(x_{1}, \ldots, x_{n}\right)\left(x_{i}: A_{i}\right)$ & $\mathbf{Y} ! \tau_{1}: A_{1}, \ldots, \tau_{n}: A_{n}$ & $\mathbf{X} ! \pi\left(\tau_{1} \ldots \tau_{n}\right)$ \\
$($ " $\pi$ " es una afirmación $)$ & $\left(\tau_{i}\right.$ es un objeto lúdico & \\
& de la forma $a_{i}: A$, & \\
& o bien de la forma & \\
& $\left.x_{i}: A\right)$ & \\
\hline
\end{tabular}

${ }^{21}$ Esta regla es la expresión (a nivel de partida) de la regla que regula la sustitución de variables en el seno de un juicio hipotético. Véase Martin-Löf 1984, pp. 9-11. 
4.3.2. Las reglas de desarrollo: significado global

SRO (Regla de inicio)

- Un diálogo formal de razonamiento inmanente comienza con una jugada de $\mathbf{P}$ por medio de la cual propone la tesis. Puede proponerse la tesis a condición de que $\mathbf{0}$ esté dispuesto a conceder ciertas afirmaciones llamadas concesiones iniciales. En este caso la tesis tiene la forma $! \alpha\left[\beta_{1}, \ldots, \beta_{n}\right]$.

- Un diálogo bajo condiciones tiene lugar si y solamente si $\mathbf{0}$ acepta tales condiciones. $\mathbf{O}$ acepta las condiciones propuestas afirmando las concesiones iniciales $! \beta_{1}, \ldots, ! \beta_{n}$. Tanto $\mathbf{O}$ como $\mathbf{P}$ deben proponer objetos lúdicos como parte de las afirmaciones que inician una partida.

- Si la tesis se propuso sin condición alguna (y no consiste en la afirmación de una proposición elemental), $\mathbf{P}$ debe asociar un objeto lúdico a la tesis.

- Si la tesis consiste en la afirmación de una proposición elemental de la forma ! $A$, la partida comienza con una jugada de $\mathbf{0}$ que solicita que $\mathbf{P}$ proponga un objeto lúdico para $A$.

- Después de la afirmación de la tesis, el oponente y el proponente eligen sucesivamente un número entero llamado rango de repetición.

El rango de repetición elegido por un jugador representa el número máximo de veces que puede desafiar o defenderse de una jugada determinada del adversario.

Toda jugada después de la elección de los rangos de repetición es: o bien un ataque, o bien una defensa.

SRli (Regla de desarrollo intuicionista)

- Cada jugada posterior a la tesis es una respuesta a una jugada anterior del adversario. Los jugadores sólo pueden defenderse del último de los ataques al que aún no han respondido.

SR1c (Regla de desarrollo clásica)

- Cada jugada posterior a la tesis es una respuesta a una jugada anterior del adversario. 
SR2 (Partida de formación para diálogos formales)

- Una partida de formación comienza con un ataque de $\mathbf{0}$ de la forma '?prop'. La partida se desarrolla siguiendo las reglas de formación hasta que se han analizado los componentes elementales de la tesis. Una vez que se ha llegado a esa etapa, se continúa el diálogo con el resto de las reglas locales y globales.

- Si una de la expresiones que constituyen la tesis no tiene regla local, entonces $\mathbf{P}$ debe proponer una definición nominal haciendo uso de un definiendum que se encuentra en una de las tablas para el significado local.

\section{SR3 (Resolución y sustitución de instrucciones)}

Terminología:

Decimos que la instrucción $I^{\kappa}(p)$ compromete al jugador $\mathrm{X}$ con $\kappa$ (donde " $I$ " es una instrucción, " $\kappa$ " un indice de instrucción descrito por las reglas locales y " $p$ " un objeto lúdico) si y solamente si:

a. $I^{\kappa}(p)$ es de la forma $L^{\forall}(p)\left(\right.$ o $\left.L^{\supset}(p)\right)$ y, en el contexto de la partida, $\mathbf{X}$ tiene la tarea de atacar el universal (o la implicación) cuyo objeto lúdico es $p$.

b. $I^{\kappa}(p)$ es una instrucción diferente de $L^{\forall}(p)\left(\right.$ o $\left.L^{\supset}(p)\right)$ y, en el contexto de la partida, $\mathbf{X}$ tiene la tarea de defender la proposición cuyo objeto lúdico es $p$.

SR3.1 (Resolución de instrucciones)

(1) Un jugador puede pedir que su adversario sustituya la instrucción con un objeto lúdico adecuado.

(2) Cuando se ha sustituido una instrucción, decimos que la instrucción se ha resuelto.

(3) Si la instrucción $I^{\kappa}(p)$ compromete al jugador $\mathbf{X}$ con $\kappa$ y es el adversario $\mathbf{Y}$ quien solicita la sustitución, el ataque es de la forma

$$
\text { ?--- / } I^{\kappa}(p)
$$

- La respuesta al ataque consiste en efectuar la sustitución requerida. 
(4) Si el jugador $\mathbf{Y}$ afirma $\pi\left[I^{\kappa}(p)\right]$, pero es al adversario $\mathbf{X}$ a quien $I^{\kappa}(p)$ compromete con $\kappa$, el ataque es de la forma

$$
\text { ? - } b-/ I^{\kappa}(p)
$$

donde $b$ es elegido por $\mathbf{X}$.

- La respuesta al ataque consiste en efectuar la sustitución requerida.

(5) En el caso de una secuencia de instrucciones de la forma $\pi\left[I_{1}\left(\ldots\left(I_{k}(p)\right) \ldots\right)\right]$, las instrucciones se resuelven del interior $\left(I_{k}(p)\right)$ al exterior $\left(I_{i}\right)$.

Un caso especial:

- La resolución de $R\urcorner(p): \perp$ conduce al abandono.

$$
\mathbf{X} ! R\urcorner(p): \perp \quad \mathbf{Y} ! I^{\kappa}: \alpha \quad \mathbf{X} ! \perp
$$

$\cdots$

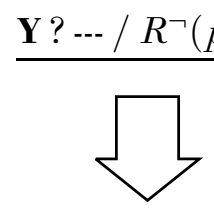

$\mathbf{X} ! \perp(n)$

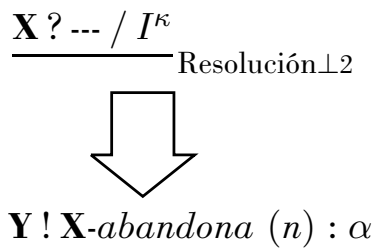

SR3.2 (Sustitución de instrucciones)

Una vez que se ha empleado el objeto lúdico $b$ para resolver la instrucción $I^{\kappa}(p)$, y si la misma instrucción ocurre nuevamente, los jugadores tienen el derecho a pedir que la instrucción vuelva a resolverse con $b$.

SR3.3 (Resolución y sustitución de funciones)

Las funciones se atacan y se defienden exactamente de la misma manera que las instrucciones.

SR4 (Regla formal modificada)

O puede atacar una proposición elemental si y sólo si él mismo aún no la ha afirmado. Sólo el oponente puede atacar proposiciones elementales. El proponente se defiende de un ataque a una proposición elemental mostrando que en el desarrollo posterior del juego el oponente 
será forzado a conceder la proposición elemental atacada, digamos en la jugada $n$. En cuanto $\mathbf{O}$ juega $n$, entonces $\mathbf{P}$ se defiende del ataque respondiendo sic $(n)$ (léase: porque tú mismo acabas de conceder en $n$ la misma proposición elemental).

\section{SR5 (Regla socrática e igualdad definicional)}

La idea básica es la siguiente:

- Las expresiones elementales de $\mathbf{P}$ que resultan de la resolución de instrucciones pueden ser atacadas por $\mathbf{0}$.

- La respuesta a estos ataques consiste en la afirmación de una igualdad definicional. Y se distinguen los casos reflexivos y no reflexivos mencionados en la discusión precedente.

- De hecho, una igualdad definicional afirmada por $\mathbf{P}$ expresa la igualdad entre un objeto lúdico, introducido por $\mathbf{0}$, y una instrucción también introducida por $\mathbf{0}$.

Esta idea será implementada en dos series de tablas, una para los casos no reflexivos y otra para los casos reflexivos. Sin embargo, las tablas no cubren los casos de transmisión de una igualdad ya establecida (la transmisión se efectúa de la forma usual, es decir, empleando reflexividad, transitividad y simetría).

Las tablas emplean las siguientes convenciones en la notación:

- La notación " $g$ " (léase funcstrion) expresa o bien una instrucción, o bien una función.

- Las expresiones sobre la línea establecen las condiciones que se requieren para que $\mathbf{P}$ pueda hacer la jugada descrita debajo de la flecha. Tales condiciones se dividen en dos conjuntos. El conjunto izquierdo, el conjunto de condiciones de ataque, describe la forma de atacar y las jugadas que permiten ese ataque. El conjunto derecho, el conjunto de condiciones de respuesta, describe las jugadas de $\mathbf{0}$ que permiten a $\mathbf{P}$ afirmar la igualdad descrita debajo de la flecha.

- La notación "Y $! a / g$ : $A$ " representa la condición "Y sustituyó $g^{\prime} \operatorname{con} a$ en $A "$.

- La expresión "type" en la afirmación " $\varphi$ : type" expresa que se trata o bien del tipo set o bien del tipo prop. 
- La expresión $g_{i} \neq \neq g_{k}$ indica que los funcstriones son sintácticamente diferentes; por ejemplo, $L^{\forall}(p)$ y $R^{\exists}(q)$.

- " $A$ " y " $A(a)$ " corresponden a expresiones elementales. " $\phi$ " es una expresión elemental de alguna de las dos formas: $A$ o $A(a)$. La resolución de un funcstrión que ocurre en $A(g)$ da como resultado una proposición elemental.

El empleo de las reglas siguientes presupone que $\mathbf{P}$ afirmó una expresión elemental o una igualdad definicional de la forma $g_{k}=a: A(b)$.

El resultado de una aplicación de la regla socrática no puede volver a recibir más ataques que los prescritos por tal regla.

SR5.1: Tabla de la regla socrática I

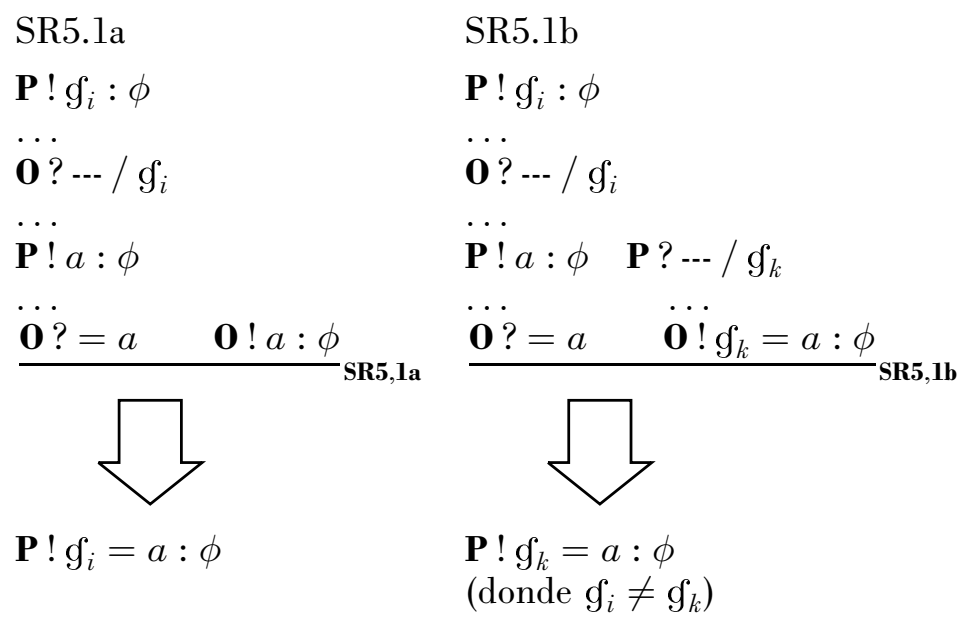

SR5.1b*

Si la afirmación de $\mathbf{P} ! a: \phi$ no es resultado de la resolución de un funcstrión, entonces la respuesta es la prescrita por SR5.1b, i.e., $\mathbf{P} ! \mathfrak{g}_{k}=a: A$.

SR5.1c

Si el resultado de aplicar alguna de las reglas SR5.1 es $A(b)$ (que es una resolución de $\left.A\left(g_{m}\right)\right)$, $\mathbf{O}$ puede lanzar un nuevo ataque contra $b$ (que ocurre en $A$ ) de la forma:

$$
\mathbf{0} ?=b^{A(b)}
$$


La respuesta de $\mathbf{P} ! g_{n}=b: D$ si $g_{n}$ fue resuelta con $b$ por $\mathbf{0}$. Si la afirmación $A(b)$ de $\mathbf{O}$ no es el resultado de una resolución de $\mathbf{0}$, la respuesta es $\mathbf{P} ! g_{m}=b: D$.

SR5.1d

Si $g=b: D$ y $\mathbf{P}$ afirma $A(b)$, entonces $\mathbf{0}$ puede requerir la identidad de $A(b)$. La respuesta es que $A(b)=A(b): \operatorname{set}(o$ prop).

SR5.2: Tabla de la regla socrática II

Las respuestas reflexivas a un ataque (sobre el uso de un objeto lúdico), tales como:

$$
\mathbf{P} ! a=a: \phi \quad \mathbf{P} ! A(b)=A(b): \text { type }
$$

asumen no solamente que $\mathbf{O}$ usó el objeto lúdico en cuestión de la misma manera, sino que el oponente lo usó en uno de los siguientes casos:

- cuando resolvió el mismo funcstrión $g_{i}$,

- cuando estableció él mismo la identidad entre $g_{i}$ y el objeto lúdico, o

- cuando afirmó $a: \phi($ o $A(b):$ type).

SR6 (Partida ganada)

- Si un jugador afirma “ $\perp$ " abandona la partida y el adversario gana. Si no hay una jugada de abandono, el jugador que hace la última jugada gana la partida. ${ }^{22}$

Partidas terminales y estrategias ganadoras

- La definiciones son las mismas que para los diálogos estándar.

${ }^{22}$ Esta regla acorta las partidas. En realidad, de acuerdo con la regla especial para $\perp$, si el jugador $\mathbf{X}$ la afirma, abandona la partida y eso le permite al adversario $\mathbf{Y}$ responder un ataque con el objeto lúdico $\mathbf{X}$-abandona $(n)$. 


\begin{tabular}{|c|c|c|c|c|c|}
\hline \multicolumn{3}{|c|}{$\mathbf{0}$} & \multicolumn{3}{|c|}{$\mathbf{P}$} \\
\hline $\begin{array}{l}\text { C1 } \\
\text { C2 }\end{array}$ & $\begin{array}{l}! C(x, y): \operatorname{set}[x: A, y: B(x)] \\
! B(x): \operatorname{set}[x: A]\end{array}$ & & & $\begin{array}{l}! d:(\forall x: A)(\exists y: B(x)) C(x, y) \supset(\exists f:(\forall x: A) \\
B(x))(\forall x: A) C(x, f(x))\end{array}$ & 0 \\
\hline 1 & $m:=1$ & & & $n:=2$ & 2 \\
\hline 3 & $! L^{\supset}(p):(\forall x: A)(\exists y: B(x)) C(x, y)$ & 0 & & $! R^{\supset}(d):(\exists f:(\forall x: A) B(x))(\forall x: A) C(x, f(x))$ & 6 \\
\hline 5 & $! v:(\forall x: A)(\exists y: B(x)) C(x, y)$ & & 3 & $? \cdots / L^{\supset}(d)$ & 4 \\
\hline 7 & $?$--- / $R^{\supset}(d)$ & 6 & & $! g:(\exists f:(\forall x: A) B(x))(\forall x: A) C(x, f(x))$ & 8 \\
\hline 9 & $?_{\mathbf{R}}$ & $\mathbf{8}$ & & $! R^{\exists}(g):(\forall x: A) C\left(x, L^{\exists}(g)(x)\right)$ & 10 \\
\hline 11 & $?$ ?-- / $L^{\exists}(g)$ & 10 & & $! R^{\exists}(g):(\forall x: A) C\left(x, g_{1}(x)\right)$ & 12 \\
\hline 13 & $?---/ R^{\exists}(g)$ & 12 & & $! g_{2}:(\forall x: A) C\left(x, g_{1}(x)\right)$ & 14 \\
\hline 15 & $! L^{\forall}\left(g_{2}\right): A$ & 14 & & $! R^{\forall}\left(g_{2}\right): C\left(x, g_{1}(a)\right)$ & 36 \\
\hline 17 & $! a: A$ & & 15 & $? \cdots / L^{\forall}\left(g_{2}\right)$ & 16 \\
\hline 23 & $! R^{\forall}(v):(\exists y: B(a)) C(a, y)$ & & 5 & $! L^{\forall}(v): A$ & 18 \\
\hline 19 & $?$ ?-- / $L^{\forall}(v)$ & 18 & & $! a: A$ & 20 \\
\hline 21 & $?=a$ & 20 & & $! L^{\forall}\left(g_{2}\right)=a: A$ & 22 \\
\hline 25 & $!\left(v_{2}\right):(\exists y: B(a)) C(a, y)$ & & 23 & $? \cdots--/ R^{\forall}(v)$ & 24 \\
\hline 27 & $! L^{\exists}\left(v_{2}\right): B(a)$ & & 25 & $?_{L}$ & 26 \\
\hline
\end{tabular}




\begin{tabular}{|l|l|l|l|l|l|}
\hline 29 & $! t_{1}: B(a)$ & & 27 & $?---/ L^{\exists}\left(v_{2}\right)$ & 28 \\
\hline 31 & $! R^{\exists}\left(v_{2}\right): C\left(a, L^{\exists}\left(v_{2}\right)\right)$ & & 25 & $? R$ & 30 \\
\hline 33 & $! R^{\exists}\left(v_{2}\right): C\left(a, t_{1}\right)$ & & 31 & $? t_{1} / L^{\exists}\left(v_{2}\right)$ & 32 \\
\hline 35 & $! t_{2}: C\left(a, t_{1}\right)$ & & 31 & $?---/ R^{\exists}\left(v_{2}\right)$ & 34 \\
\hline 37 & $?--/ R^{\forall}\left(g_{2}\right)$ & 36 & & $! t_{2}: C\left(a, g_{1}(a)\right)$ & 38 \\
\hline 39 & $?--/ g_{1}(a)$ & 38 & & $! t_{2}: C\left(a, t_{1}\right)$ & 40 \\
\hline 41 & $?=t_{2}$ & 40 & & $! R^{\exists}\left(v_{2}\right)=t_{2}: C\left(a, t_{1}\right)$ & 42 \\
\hline 43 & $?=a^{C\left(a, t_{1}\right)}$ & 42 & & $L^{\exists}\left(v_{2}\right)=t_{1}: B(a)$ & 44 \\
\hline 45 & $?=C\left(a, t_{1}\right):$ type & 44 & & $! C\left(a, L^{\exists}\left(v_{2}\right)\right)=C\left(a, t_{1}\right):$ set & 48 \\
\hline 47 & $C\left(a, t_{1}\right):$ type & & $\mathrm{C} 1$ & $! a: A, t_{1}: B(a)$ & 46 \\
\hline
\end{tabular}

Jugada 3: después de que $\mathbf{P}$ postula la tesis y se han establecido los rangos de repetición, $\mathbf{O}$ comienza atacando la implicación material.

do sobre la justificación que la respalda. $\mathbf{P}$ responde afirmando que el objeto lúdico escogido es el mismo que eligió $\mathbf{O}$ cuando resolvió $L^{\forall}\left(g_{2}\right)$.

JUGADA 4: $\mathbf{P}$ contraataca exigiendo la resolución de $L^{\supset}(d)$.

JugADA 9: $\mathbf{O}$ puede ahora elegir entre la derecha y la izquierda, el presente juego se desarrolla a partir de la segunda opción de $\mathbf{0}$.

JUGADAS 21-22: introducen la primera igualdad definicional del juego: $\mathbf{P}$ afirmó $a: A$ en la jugada 18. Dado que es una expresión elemental, $\mathbf{0}$ la ataca preguntan-

JUGADAS 41-48: requieren cierto cuidado. En efecto, no le basta a $\mathbf{P}$ defender la jugada 42 con la igualdad $L^{\exists}\left(v_{2}\right)=t_{1}: B(a)$. $\mathbf{P}$ tiene también que justificar $C\left(a, t_{1}\right)$. Con el fin de llevar a cabo la defensa, $\mathbf{P}$ recurre a la formación de $C\left(a, t_{1}\right)$ que resulta de la concesión inicial $C(x, y): \operatorname{set}[x: A, y: B(x)](\mathrm{Cl})$. Esto le permite establecer la igualdad crucial de la victoria $C\left(a, L^{\exists}\left(v_{2}\right)\right)=C\left(a, t_{1}\right)$ : set. 
5. Presentación breve de un ejemplo célebre: la versión intensional del axioma de elección

Per Martin-Löf (1984, pp. 50-51) mostró que puede demostrarse la versión intensional del así llamado axioma de elección (la demostración le valió el premio Kolmogorov). Clerbout y Rahman (2015) realizaron un estudio meticuloso y detallado de la interpretación dialógica de esa prueba.

Desde el punto de vista dialógico, la idea fundamental de la demostración es que la estrategia ganadora de $\mathbf{P}$ se desarrolla a partir de jugadas que copian las elecciones del oponente que resultan de las obligaciones contraídas por $\mathbf{0}$. Para ser más precisos, $\mathbf{P}$ defenderá el consecuente de la implicación principal ejerciendo el derecho a copiar las elecciones de $\mathbf{0}$. Por razones de espacio sólo presentamos aquí uno de los juegos posibles que definen el núcleo de la estrategia ganadora. Véase la tabla de las pp. 84 y 85.

\section{Conclusiones}

La igualdad definicional tiene un rol central en la constitución de un conjunto desde el punto de vista de la TCT, y en el contexto de la lógica, tal forma de igualdad hace explícita la coordinación de los procesos inferenciales analíticos y sintéticos que otorgan las normas de significado para el tipo de las constantes lógicas. La contribución dialógica al análisis desarrollado por la TCT es hacer explícito que los aspectos normativos de la lógica, si ésta se explica mediante la interpretación dialógica que sugiere Martin-Löf, provienen de un nivel básico de interacción en el que la igualdad definicional expresa el concepto fundamental del razonamiento formal.

Un corolario del presente estudio que consideramos digno de mencionar es que proporciona una comprensión más profunda del enfoque dialógico sobre cómo vincular la noción de armonía en la TCT (Rahman y Redmond 2016) con el concepto de razonamiento formal. En efecto, dado que Rahman y Redmond (2016) mostraron que la noción de armonía en la TCT — que se desprende de las reglas de igualdad definicional - está relacionada en el cuadro dialógico con la noción de independencia de los jugadores y dado que el presente estudio muestra que la igualdad definicional es el producto de la regla socrática que prescribe las jugadas de espejo, podemos entonces concluir la siguiente caracterización de la armonía dialógica: la armonía es el producto de las reglas de interacción que deben estar formuladas de tal manera que coordinen la independencia de los 
jugadores (propia del significado local) con la igualdad prescrita por la regla socrática. Esta forma de armonía es la que establece la norma dialógica de un razonamiento formal. ${ }^{23}$

\section{BIBLIOGRAFÍA}

Clerbout, N., 2014a, "First-Order Dialogical Games and Tableaux", Journal of Philosophical Logic, vol. 43, no. 4, pp. 785-801.

$\longrightarrow$ - 2014b, La Sémantique dialogique: Notions fondamentales et éléments de metathéorie, College Publications, Londres.

Clerbout, N. y S. Rahman, 2015, Linking Game-Theoretical Approaches with Constructive Type Theory: Dialogical Strategies as TCT-Demonstrations, Springer, Dordrecht.

- 2013, "On Dialogues, Predication and Elementary Sentences", Revista de Humanidades de Valparaíso, vol. 1, no. 2, pp. 7-46.

Granström, J., 2011, Treatise on Intuitionistic Type Theory, Springer, Dordrecht.

Keiff, L., 2009, "Dialogical Logic", en E.N. Zalta (ed.), The Stanford Encyclopedia of Philosophy, disponible en $<$ http://plato.stanford.edu/entries/ logic-dialogical>, [última consulta: 1 de septiembre de 2016].

$\longrightarrow$, 2007, Le pluralisme dialogique. Approches dynamiques de l'argumentation formelle, tesis de doctorado, Universidad de Lille, Lille.

Lorenz, K., 2010a, Logic, Language and Method: On Polarities in Human Experience, De Gruyter, Berlín.

- 2010b, Philosophische Variationen: Gesammelte Aufsätze unter Einschluss gemeinsam mit Jürgen Mittelstraß geschriebener Arbeiten zu Platon und Leibniz, De Gruyter, Berlín.

—_, 2008, Dialogischer Konstruktivismus, De Gruyter, Berlín.

,2001, "Basic Objectives of Dialogue Logic in Historical Perspective", Synthese, vol. 127, nos. 1-2, New Perspectives in Dialogical Logic, pp. 255-263.

Lorenzen, P. y K. Lorenz, 1978, Dialogische Logik, Wissenschaftliche Buchgesellschaft, Darmstadt.

${ }^{23}$ Los resultados presentados aquí se obtuvieron en el marco del proyecto Fondecyt regular no. 1141260 (CONICYT) y en el marco de los programas de investigación Argumentation, Décision, Action (ADA) de la Maison Européenne des Sciences de l'Homme et de la Société - USR 318, el eje transversal de investigación Argumentation del laboratorio UMR 8163: STL y el proyecto ANR SEMAINO.

Nuestro profundo agradecimiento a los siguientes colegas por la enriquecedora y fructífera discusión en torno al tema: Ansten Klev (Praga), Gildas Nzokou (Libreville, Gabón), Clément Lion (Lille), Steephe Rossy (Lille), Zoe McConaughey (Lille), Göran Sundholm (Leiden), Johan Granström (Zúrich) y Laurent Keiff (Lille).

Asimismo, queremos agradecer los valiosísimos informes que presentaron los árbitros anónimos sobre una versión anterior de este mismo artículo, los cuales nos permitieron mejorarlo profundamente. 
Marion, M. y H. Rückert, 2015, “Aristotle on Universal Quantification: A Study from the Perspective of Game Semantics", History and Philosophy of Logic, vol. 37, no. 3, pp. 201-229.

Martin-Löf, P., 1996, "On the Meanings of the Logical Constants and the Justifications of the Logical Laws", Nordic Journal of Philosophical Logic, vol. 1, no. 1, pp. 11-60.

— 1984, Intuitionistic Type Theory. Notes by Giovanni Sambin of a Series of Lectures Given in Padua, June 1980, Bibliopolis, Nápoles.

Nordström, B., K. Petersson y J.M. Smith, 1990, Programming in MartinLöf's Type Theory: An Introduction, Oxford University Press, Oxford.

Peregrin, J., 2014, Inferentialism: Why Rules Matter, Palgrave MacMillan, Nueva York.

Prawitz, D., 1965, Natural Deduction: A Proof-Theoretical Study, Almqvist and Wiksell, Estocolmo.

Rahman, S., 2012, "Negation in the Logic of First Degree Entailment and Tonk: A Dialogical Study", en S. Rahman, G. Primiero y M. Marion (comps.), The Realism-Antirealism Debate in the Age of Alternative Logics, Springer, Dordrecht, pp. 213-250.

—_ 1993, Über Dialogue, Protologische Kategorien und andere Seltenheiten, Peter Lang, Fráncfort/París/Nueva York.

Rahman, S. y N. Clerbout, 2014, "Constructive Type Theory and the Dialogical Turn - A New Approach to Erlangen Constructivism”, en J. Mittelstrass y C. von Bülow (comps.), Dialogische Logik, Mentis, Münster, pp. 91-148.

—_ 2013, "Constructive Type Theory and the Dialogical Approach to Meaning", The Baltic International Yearbook of Cognition, Logic and Communication, vol. 8, Games, Game Theory and Game Semantics, pp. 1-72, disponible en <http://www.thebalticyearbook.org >.

Rahman, S., N. Clerbout y R. Jovanovic, 2016, "Knowledge and Its Game Theoretical Foundations: The Challenge of the Dialogical Approach to Constructive Type Theory", en J. Redmond, O. Pombo y A. Nepomuceno (comps.), Epistemology, Knowledge and the Impact of Interaction, Springer, Dordrecht, pp. 23-73.

—_ 2015, “The Dialogical Take on Martin-Löf's Proof of the Axiom of Choice", South American Journal of Logic, vol. 1, no. 1, pp. 179-208.

Rahman, S., N. Clerbout y L. Keiff, 2009, "On Dialogues and Natural Deduction", en S. Rahman y G. Primiero (comps.), Acts of Knowledge: History, Philosophy and Logic. Essays Dedicated to Göran Sundholm, College Publications, Londres, pp. 301-336.

Rahman, S., N. Clerbout y Z. McConaughey, 2014, "On Play-Objects in Dialogical Games. Towards a Dialogical Approach to Constructive Type Theory", en P. Allo y B. van Kerkhove (comps.), Modestly Radical or Radically Modest. Festschrift for Jean-Paul van Bendegem, College Publications, Londres, pp. 127-154.

Rahman, S. y L. Keiff, 2010, "La Dialectique entre Logique et Rhétorique", Revue de Métaphysique et de Morale, vol. 66, no. 2, pp. 149-178. 
Rahman, S. y L. Keiff, 2005, "On How to Be a Dialogician", en D. Vanderveken (comp.), Logic, Thought, and Action, Kluwer, Dordrecht, pp. 359-408.

Rahman, S., Z. McConaughey y M. Crubellier, 2016, “Aristotelian Syllogism as Dialogical Games", inédito.

Rahman, S. y J. Redmond, 2016, “Armonía dialógica: tonk, teoría constructiva de tipos y reglas para jugadores anónimos", Theoria, vol. 31, no. 1, pp. 27-53.

- 2015, "A Dialogical Frame for Fictions as Hypothetical Objects", Filosofía Unisinos, vol. 16, no. 1, pp. 2-21.

Rahman, S. y T. Tulenheimo, 2009, "From Games to Dialogues and Back: Towards a General Frame for Validity", en O. Majer, A. Pietarinen y T. Tulenheimo (comps.), Games: Unifying Logic, Language and Philosophy, Springer, Dordrecht, pp. 153-208.

Ranta, A., 1994, Type-Theoretical Grammar, Clarendon Press, Oxford.

- 1988, "Propositions as Games as Types", Synthese, vol. 76, pp. 377395.

Redmond, J. y M. Fontaine, 2011, How to Play Dialogues: An Introduction to Dialogical Logic, College Publications, Londres.

Rückert, H., 2011, Dialogues as a Dynamic Framework for Logic, College Publications, Londres.

Sundholm, G., 2013, "Inference and Consequence in an Interpreted Language", presentación en el Workshop Proof Theory and Philosophy, Gröningen, 3-5 de diciembre de 2013.

— 2009, "A Century of Judgment and Inference: 1837-1936", en L. Haaparanta (comp.), The Development of Modern Logic, Oxford University Press, Oxford, pp. 263-317.

- 2001, "A Plea for Logical Atavism", en O. Majer (comp.), The Logica Yearbook 2000, Filosofia, Praga, pp. 151-162.

_ 1998, "Inference versus Consequence", en T. Childers (comp.), The Logica Yearbook 1997, Filosofia, Praga, pp. 26-36.

- 1997, "Implicit Epistemic Aspects of Constructive Logic", Journal of Logic, Language, and Information, vol. 6, no. 2, pp. 191-212.

— , 1986, "Proof-Theory and Meaning", en D. Gabbay y F. Guenthner (comps.), Handbook of Philosophical Logic, vol. 3, Reidel, Dordrecht, pp. 471-506.

— 1983, "Constructions, Proofs, and the Meaning of Logical Constants", Journal of Philosophical Logic, vol. 12, no. 2, pp. 151-172.

Recibido el 6 de julio de 2016; revisado el 28 de febrero de 2017; aceptado el 7 de abril de 2017. 